\title{
EL USO DEL NEUROMARKETING Y DEL MARKETING SENSORIAL EN LOS EVENTOS: UN ESTUDIO DE CASO
}

\section{THE USE OF NEUROMARKETING AND SENSORY MARKETING AT EVENTS: A CASE STUDY}

\author{
Guerra Serrano, Alberto ${ }^{1}$ \\ Gomes-Franco e Silva, Flávia² \\ Universidad Rey Juan Carlos \\ aguerraserrano@hotmail.com \\ flavia.gomes@urjc.es
}

Material original autorizado para su primera publicación en la revista académica REDMARKA. Revista Digital de Marketing Aplicado.

https://doi.org/10.17979/redma.2017.01.018.4857

Recibido: 17 Mayo 2017

Aceptado: 21 Junio 2017

Material original autorizado para su primera publicación en la revista académica REDMARKA. Revista Digital de Marketing Aplicado.

\section{Resumen}

\footnotetext{
${ }^{1}$ Máster en Protocolo, Comunicación y Organización Integral de Eventos por la Universidad Rey Juan Carlos (España). Graduado en Publicidad y Relaciones Públicas por la Universidad Complutense (España). Investigador en la URJC. Planificador de eventos, diseñador de escenarios, webs, montajes de vídeo, newsletters y cualquier diseño requerido.

2 Doctora y Máster en Ciencias de la Comunicación por la Universidad Rey Juan Carlos (España). Licenciada en Periodismo por la Universidade Federal de Goiás (Brasil). Docente de programas de grado y post-grado de la Universidad Rey Juan Carlos (URJC), vinculada a la Facultad de Ciencias Jurídicas y Sociales. Miembro investigador del Proyecto Nacional de I+D "El sistema de investigación en España sobre prácticas sociales de comunicación. Mapa de proyectos, grupos, líneas, objetos de estudio y métodos" (España).
} 


\section{EL USO DEL NEUROMARKETING Y DEL MARKETING SENSORIAL EN LOS EVENTOS: UN ESTUDIO DE CASO}

Guerra Serrano, Alberto

A partir de las últimas décadas del pasado siglo se han implementado diversas técnicas de marketing derivadas de estudios realizados en diferentes áreas del conocimiento, como la psicología o la neurociencia. Se identifica, a raíz de dichas investigaciones, una serie de elementos que condicionan la toma de decisiones de los consumidores sin que estos sean conscientes de que están bajo la influencia de factores externos que les motivan a optar por una marca en detrimento de otras. El presente estudio propone un acercamiento a la utilización de técnicas de neuromarketing y marketing sensorial en un evento de carácter promocional realizado por la empresa japonesa de fabricación de automóviles Suzuki en el mes de noviembre de 2015, en el municipio de Boadilla del Monte (Madrid, España). Se realiza un estudio de caso mediante la técnica de la observación directa y, de manera complementaria, se aplica una encuesta breve a algunos de los invitados al evento a modo de feedback. Los resultados del análisis apuntan a un efecto potenciador de la experiencia vivida en el evento debido al empleo de técnicas que pretenden estimular los sentidos y actuar de manera positiva en el recuerdo de la marca. El estudio incide en la importancia de ahondar en las investigaciones acerca del uso de las diferentes técnicas de neuromarketing y marketing sensorial en los eventos, objeto escasamente analizado en el ámbito académico.

Palabras clave: Neuromarketing, Marketing sensorial, Marketing de experiencias

\section{Abstract}

From the last decades of the twentieth century it has implemented various marketing techniques derived from studies conducted in different areas of knowledge, such as psychology or neuroscience. Because of these investigations, it has been identified several elements that influence the decision making of consumers without them being aware that they are under the influence of external factors that motivate them to choose one brand over others. This study 


\section{EL USO DEL NEUROMARKETING Y DEL MARKETING SENSORIAL EN LOS EVENTOS: UN ESTUDIO DE CASO}

Guerra Serrano, Alberto

proposes an approach to the use of neuromarketing and sensory marketing techniques in a promotional event by the Japanese automotive multinational corporation Suzuki in November 2015 in the municipality of Boadilla del Monte (Madrid, Spain). A case study is performed using the technique of direct observation and, in a complementary way, a brief survey has been applied to some of the event guests as a feedback. The results of the analysis show a potentiation effect of the lived experience in the event due to the use of techniques that aim to stimulate the senses and act positively in the memory of the brand. The study highlights the importance of deepening research on using different techniques of neuromarketing and sensory marketing in events, object barely analyzed in academic environment.

Keywords: Neuromarketing, Sensory marketing, Experience marketing

\section{Introducción}

La situación económica actual y el nivel de competitividad del mercado han hecho necesario un cambio en la forma en la que un producto o servicio es creado, vendido o presentado. Los clientes buscan nuevas experiencias y las empresas, por su parte, buscan sorprender. A partir de la década de 1970, investigadores como Bloch et al. (1986) se propusieron descubrir qué elementos motivaban a los consumidores a decantarse por unos productos en lugar de otros. Tauber (1972) ya había planteado la posibilidad de que las personas prefirieran ciertos productos independientemente de un razonamiento lógico, sugiriendo que existen diversas razones subyacentes que tienen lugar en el cerebro de los consumidores sin que estos sean conscientes de ellas.

Las emociones, sensaciones y percepciones juegan un papel fundamental en la toma de decisiones de los consumidores y en los procesos de recuerdo de una marca. Muchas investigaciones en el campo del marketing se centran en el momento justo de la toma de decisiones (Burnkrant y Cousineau, 1975). 


\section{EL USO DEL NEUROMARKETING Y DEL MARKETING SENSORIAL EN LOS EVENTOS: UN ESTUDIO DE CASO}

Guerra Serrano, Alberto

Conscientes de que deben diferenciar sus productos y conocer más acerca de los consumidores, sus inquietudes y las verdaderas razones que les mueven a comprar, cada vez más las marcas buscan soluciones a través de la ciencia recurriendo a las aplicaciones prácticas del neuromarketing y del marketing sensorial.

Las empresas afrontan a diario la dificultad que implica sorprender a los consumidores debido a la gran variedad de productos de diferente índole existentes en el mercado. Esta coyuntura ha estimulado la realización de investigaciones sobre la percepción, las cuales aportan al marketing una serie de técnicas y herramientas que logran comunicarse de manera más eficaz con los públicos objetivos.

La búsqueda de nuevas experiencias constituye uno de los objetivos principales de las empresas debido a la necesidad de diferenciarse de sus competidores. A raíz de las investigaciones en neuromarketing, se ha procurado actuar de una manera más efectiva en el recuerdo de los consumidores, su comportamiento y su actitud a la hora de efectuar una compra (Morrin et al., 2011). De este modo, aparece en escena el llamado marketing sensorial.

El marketing sensorial o marketing de experiencias hace hincapié en el estudio y la aplicación de los cinco sentidos en relación con la investigación de mercados y con el marketing. En las últimas décadas, diversos estudios han demostrado que trabajar el gusto, el olfato, el oído, el tacto y la vista en todo el universo que rodea al producto puede mejorar el impacto y las tasas de recuerdo obtenidas por los consumidores (Abril et al., 2011). Es por ello que muchas empresas focalizan sus esfuerzos en la mejora de la experiencia de compra y en la intensificación del uso de los sentidos durante la misma.

Las técnicas resultantes del neuromarketing y del marketing sensorial pueden ser identificadas en los puntos de venta de las distintas marcas. Se observa una proliferación de las pantallas LED, el uso de la música con un volumen elevado o los diferentes tipos de fragancias y ambientadores. 


\section{EL USO DEL NEUROMARKETING Y DEL MARKETING SENSORIAL EN LOS EVENTOS: UN ESTUDIO DE CASO}

Guerra Serrano, Alberto

Otro de los campos que ha experimentado un gran cambio a partir de la evolución de dichas técnicas ha sido la publicidad. Aunque el $83 \%$ de la información es recibida a través de los ojos (Lindstrom, 2005), solo una pequeña cantidad se mantiene en nuestra mente con el paso del tiempo. Por ello, la publicidad trata de poner énfasis en el intento de evocar a los sentidos en sus anuncios y campañas.

En este contexto, es imprescindible hacer referencia a uno de los estandartes de las marcas a la hora de presentar y vender sus productos: el evento. En él radica una de las grandes ventajas respecto a la publicidad y a los puntos de venta tradicionales. Se trata de la flexibilidad. Los eventos son susceptibles a la implementación de acciones que estimulen los sentidos, mejorando el recuerdo del producto y la imagen de marca. Acorde con las técnicas de neuromarketing y marketing sensorial, se utilizan elementos como las pantallas digitales para estimular el sentido de la vista, la música para estimular el sentido del oído o la recreación de olores para estimular el sentido del olfato. Las sensaciones provocadas en los participantes de un evento pueden ser la clave para consolidar un recuerdo que podrá redundar en una decisión de compra.

El presente estudio tiene por objeto el análisis de un evento en el que se han aplicado técnicas asociadas al neuromarketing y al marketing sensorial. Se analizan los elementos físicos del evento, observando de qué manera los sentidos son potenciados durante el acontecimiento. Para ello, se ha elegido un evento real para proceder al análisis in situ del mismo con el fin de contestar preguntas como: ¿qué elementos pueden ser utilizados para potenciar los sentidos durante un evento?, ¿la aplicación de técnicas de neuromarketing y marketing sensorial en los eventos realmente ayuda a mejorar la experiencia vivida por los invitados?, ¿qué ventajas presentan dichas técnicas? o ¿cuál es la percepción de los participantes respecto a un evento construido sobre la base del neuromarketing y el marketing sensorial? 


\section{EL USO DEL NEUROMARKETING Y DEL MARKETING SENSORIAL EN LOS EVENTOS: UN ESTUDIO DE CASO}

Guerra Serrano, Alberto

\subsection{Definiciones y bases del neuromarketing}

El término neuromarketing fue acuñado por Ale Smidts en el año 2002, quien lo definió como la utilización de las teorías y métodos de la neurociencia cognitiva para planificar, ejecutar y medir las actividades del marketing (Lewis y Bridger, 2005). Braidot (2009, p. 16) se refiere al neuromarketing como una "disciplina de avanzada, que investiga y estudia los procesos cerebrales que explican la conducta y la toma de decisiones de las personas en los campos de acción del marketing tradicional".

Por lo tanto, el neuromarketing aplica las teorías de la neurociencia para conocer el proceso de compra de los consumidores y, así, lograr un incremento del índice de ventas. Para el experto en management Peter F. Drucker, "el objetivo principal del Neuromarketing es el de decodificar procesos que forman parte en la mente del consumidor, de manera que podamos descubrir sus deseos, ambiciones y causas ocultas en sus opciones de compra" (Boricean, 2009).

El neuromarketing surge oficialmente en junio de 2002, cuando el Brighthouse Institute for Thought Sciences publicó una investigación que empleaba técnicas de Imágenes por Resonancia Magnética funcional (fMRI), un procedimiento seguro y no invasivo, cuya misión era la identificación de patrones de actividad cerebral. Con esto intentaban revelar la manera en la que un consumidor evaluaba un producto y su decisión de compra. "Thought Sciences marketing analysts use this information to more accurately measure consumer preference, and then apply this knowledge to help marketers better create products and services and to design more effective marketing campaigns" (Brighthouse Institute for Thought Sciences, 2002).

Con el desarrollo del neuromarketing, se han ido averiguando detalles relevantes para la venta de productos. Según Braidot (2009, p. 10), "prácticamente el $90 \%$ de las decisiones que tomamos como consumidores tienen su origen en modalidades que se alojan en las profundidades de nuestra mente y que nosotros mismos desconocemos". 


\section{EL USO DEL NEUROMARKETING Y DEL MARKETING SENSORIAL EN LOS EVENTOS: UN ESTUDIO DE CASO}

Guerra Serrano, Alberto

Lee et al. (2007) ven el neuromarketing como un campo de estudio válido y que va más allá de la simple venta de productos. Por su parte, Hubert y Kenning (2008) lo entienden como una actividad propia de los negocios y no exactamente como un campo de estudio diferenciado.

En esta línea, De Andreis (2012) pondera que "el Neuromarketing se origina en la neurometría, siendo esta una parte de las diferentes disciplinas, dentro de las Neurociencias, que a su vez también son partícipes del proceso de estudio y análisis de Neuromarketing". Sus áreas de origen son: neuroanatomía, neurología, neuropsicología, neuroendocrinología, neurociencias cognitivas y neuroeconomía o neurofinanzas.

El neuromarketing se centra en el estudio del cerebro de los consumidores, siendo este, según Manzano et al. (2012), un "sistema abierto y en continuo cambio y formación de nuevas redes neuronales, desarrolladas por las propias experiencias y aprendizajes". Además, el cerebro está dividido en tres sistemas y cada uno realiza funciones diferentes.

En la práctica, las herramientas más utilizadas por el neuromarketing para llevar a cabo sus estudios y conseguir mejores resultados son la fMRI y la Electroencefalografía (EEG). Puede emplearse, además, el eye tracking (Duchowski, 2007) con el fin de mejorar su funcionamiento.

La ventaja de la fMRI es su precisión: "According to No Lie MRI (an American company), current accuracy of fMRI tests is over $90 \%$, but it is estimated to be 99\% once product development is complete" (Blakemore, 2010). Sin embargo, Kenning et al. (2009) relatan que la mayoría de las empresas que afirman trabajar con métodos propios de la neurociencia aplican tan solo los conocimientos neurocientíficos provenientes de estudios ya realizados, sin aportar nuevas investigaciones o hallazgos.

Por su parte, la EEG es un procedimiento no invasivo dotado de unos sensores llamados electroencefalográficos que son capaces de capturar las señales eléctricas de frecuencias muy bajas producidas por la actividad cerebral (Sebastian, 2013). Sobre esta técnica, Pradeep (2008) señala que: “Many areas 


\section{EL USO DEL NEUROMARKETING Y DEL MARKETING SENSORIAL EN LOS EVENTOS: UN ESTUDIO DE CASO}

Guerra Serrano, Alberto

of the brain are responsible for several functions, and because of this, the EEG technique has full-brain coverage to know exactly which regions are operating simultaneously and in concert in response to a specific stimulus".

Una de las teorías actuales asociadas al neuromarketing es la que aporta Braidot (2009). El autor afirma que existe un "botón de compra" en el cerebro de los consumidores. Cuando este botón es activado, el cliente no utiliza la mente racional, por lo que estará dispuesto a adquirir el producto sin inconvenientes. La teoría del botón de compra se basa en el vínculo que se va creando entre la marca y el cliente. Para Braidot, la mejor manera de construir una gran estrategia de marketing es focalizar en la venta por impulso a corto plazo y crear vínculos estrechos con la marca a largo plazo, lo que redunda en la fidelización.

\subsection{Definiciones y bases del marketing sensorial}

Krishna et al. (2010) definen el marketing sensorial como el marketing que involucra los sentidos de los consumidores y actúa en su comportamiento. Manzano et al. (2011) lo interpretan como una comunicación enfocada en servir a los cinco sentidos del cliente. Por su parte, Lindstrom (2008) asegura que los consumidores se han vuelto inmunes a los estímulos que les aporta la publicidad tradicional, por lo que es vital utilizar la neurociencia para diseñar estrategias eficaces de marketing.

En los últimos años, se observa un cambio en la realidad del marketing. Schmitt (1999) afirma que los consumidores están experimentando el paso del marketing tradicional al sensorial gracias al surgimiento de nuevas técnicas que se centran en la oferta de experiencias. Sobre esta cuestión, Álvarez del Blanco (2011) señala que en los próximos años las marcas implementarán una serie de estrategias focalizadas en los sentidos que aportarán experiencias distintas y únicas a los consumidores.

Uno de los temas en los que más se ha centrado el marketing sensorial es en el sistema de aprendizaje de los consumidores. Según Manzano et al. (2012), "a partir del cerebro se interpreta el mundo exterior por medio del

REDMARKA UIMA-Universidad de A Coruña - CIECID

Año X, Número 18, (2017), v I pp. 21-47

http://www.redmarka.net/ ISSN 1852-2300 


\section{EL USO DEL NEUROMARKETING Y DEL MARKETING SENSORIAL EN LOS EVENTOS: UN ESTUDIO DE CASO}

Guerra Serrano, Alberto

continuo procesamiento de la información recibida a través de los sentidos". A continuación, la información recogida es analizada por las neuronas y, una vez filtrada por las experiencias archivadas en la memoria, se consigue el aprendizaje.

Para lograr crear percepciones y conductas sobre los consumidores de una manera más eficaz y estimular sus sentidos de una forma personalizada, las marcas crean sus distintas firmas sensoriales. Una firma sensorial es un olor, un sonido o un elemento visual relacionado con el diseño que diferencia a una marca de otra (Manzano et al., 2012). Investigar, definir, desarrollar e implementar una firma sensorial no es un proceso especialmente complejo. Conseguir que realmente se convierta en un símbolo de comunicación de la marca hacia el cliente, sí.

\subsection{La vista y los demás sentidos}

La vista es el sentido más desarrollado en los seres humanos y el que más información recoge. Especialistas como Bone y Ellen (1992) afirman que las imágenes han demostrado ayudar en el procesamiento de la información de un producto, facilitando la memorización y la persuasión. Por el contrario, la ausencia de imágenes tiene un efecto negativo en la persuasión (Petrova y Cildiani, 2005).

Balcetis y Dunning (2006) constataron que las personas interpretan la información visual en función de lo que más les interesa. Además, existe un proceso de selección inconsciente de lo que se considera más relevante en un momento dado, lo que genera la llamada por Simons y Chabrics (1999) ceguera por atención, que ocurre cuando una persona que está visualizando un objeto o una escena completa deja de ver los elementos no relacionados con lo que llama su atención.

Estas investigaciones son relevantes a la hora de crear o modificar los aspectos visibles que construyen una marca. Por lo tanto, elementos como la marca gráfica, el diseño de las tiendas, la política de comunicación y la política 


\section{EL USO DEL NEUROMARKETING Y DEL MARKETING SENSORIAL EN LOS EVENTOS: UN ESTUDIO DE CASO}

Guerra Serrano, Alberto

de empleados pueden ser aspectos clave en la construcción y consolidación de la firma visual de la marca.

No hemos de olvidar que la luz forma parte del ambiente creado en torno a una marca, siendo este uno de los aspectos principales en su comunicación visual. Según Rieunier (2002), las diferencias entre la luz natural y la ambiental provienen de la fuente de luz y su intensidad. En este sentido, a mayor uso de luz artificial, mayores serán la sensación de calidez y el tiempo que un cliente permanezca en una tienda.

Para el sentido visual, toman especial importancia las tecnologías a la hora de mostrar vídeos, publicidad o la marca gráfica. Según Matthews (2008), las pantallas más utilizadas son CRT, LCD o LED, DLP y plasma. Todo el contenido volcado en estas pantallas y que puede ser controlado desde un único punto es conocido como digital signage o señalización digital. De acuerdo con el III Estudio Anual de Digital Signage (IAB Spain, 2015), el 87\% de los consumidores encuestados consideran que una marca que se anuncia en pantallas digitales es moderna e innovadora. El estudio señala que, ante los soportes digitales utilizados por las marcas, la pantalla táctil es el que dispone de mayor interactividad y el más utilizado por los usuarios, superando con creces los códigos QR, el bluetooth o las redes sociales. En definitiva, el digital signage está cobrando especial relevancia a la hora de presentar anuncios (47\%), de informar de los servicios que se ofrecen en el lugar donde se encuentra el consumidor $(30 \%)$ o en eventos culturales y de ocio $(27 \%)$, de lo que se infiere que es recomendable el uso de dicha tecnología en eventos.

Aparte de la vista, los demás sentidos son también contemplados en diversas estrategias de marketing. Su papel en la creación de una identidad fuerte para la marca es clave para aumentar el recuerdo y mejorar la imagen de la misma. Es lo que Lindstrom (2005) apoda como "Branding 5D", es decir, estudiar y potenciar la estrategia a utilizar en referencia a cada uno de los sentidos humanos. 


\section{EL USO DEL NEUROMARKETING Y DEL MARKETING SENSORIAL EN LOS EVENTOS: UN ESTUDIO DE CASO}

Guerra Serrano, Alberto

Una marca, como se ha mencionado, posee una firma visual que la identifica y la diferencia de las demás a partir de una serie de elementos visuales. Igualmente, este hecho puede suceder con el resto de los sentidos. En el caso del sentido auditivo, los consumidores pueden reconocer a una marca por la canción utilizada en un anuncio, por algún tipo de ruido característico o por la voz de algún personaje famoso ligado a las campañas de esa marca. Dos ejemplos de buen marketing sonoro son Nokia, con el tono para llamadas Nokia Tune, o Intel, con su melodía Intel Inside. En el caso del sentido olfativo, un olor propio de un producto actúa de manera identificativa y asociativa. El mismo caso se da con el sentido del tacto, como puede ser el tacto de una patata frita ondulada que recuerda a cierta marca. Por último, cabe destacar que el gusto cobra especial relevancia en las marcas relacionadas con el sector alimentario.

Para Zucco (2003), el olfato es un sentido muy importante para las marcas, puesto que las personas son capaces de reconocer un olor al que fueron expuestas con anterioridad. Este hecho tiene un gran valor para las marcas debido al poder recordatorio que posee el sentido olfativo. Si logramos relacionar un olor específico a una marca y conseguimos exponer a una persona a ese olor en un momento determinado, con el paso del tiempo, al ser expuesta otra vez al mismo olor, podríamos conseguir el recuerdo de marca y, consecuentemente, el recuerdo de las experiencias vividas en el momento en el que fue expuesta por primera vez a ese olor.

La correcta elección del tipo de olor que se asociará a una marca, a un producto o a un evento puede influir de forma directa en una valoración positiva de los mismos. Según Spangenberg et al. (1996), un olor que sea agradable para el consumidor puede conseguir alargar su estancia en el punto de venta, mejorando su valoración y aumentando su intención de compra. Manzano et al. (2012) explican que el sentido olfativo se caracteriza por su lentitud y, a la vez, por su persistencia. Una persona tarda diez veces más en percibir el aroma de un objeto que en visualizarlo, aunque esa percepción, a su vez, tardará mucho más en desaparecer. 


\section{EL USO DEL NEUROMARKETING Y DEL MARKETING SENSORIAL EN LOS EVENTOS: UN ESTUDIO DE CASO}

Guerra Serrano, Alberto

Celier (2004) sostiene que la mayor efectividad de un punto de venta se consigue con una gran capacidad de manejar la subjetividad del público objetivo. La música, por ejemplo, llega a cualquier rincón del punto de venta y es una de las partes que compone la atmósfera creada por la marca, siendo un elemento de gran importancia a la hora de actuar en la percepción.

El gusto, por su parte, se encuentra muy condicionado por el resto de sentidos: el sabor del limón se asocia con el color amarillo y el sabor naranja con el color homónimo. También influye en el sabor el sentido del tacto, debido a las diferentes texturas de los productos alimenticios, y el de la audición, puesto que el sonido condiciona el gusto y puede crear una característica diferencial en cada producto.

Finalmente, el tacto tiene una serie de características diferenciales respecto a los demás sentidos. La primera diferencia es que este necesita de un contacto directo, sin intermediarios, con el producto. Este contacto se produce a partir de una predisposición activa y voluntaria por parte de los consumidores. Además, el tacto actúa en combinación con otros sentidos, generando experiencias multisensoriales.

\subsection{El neuromarketing y el marketing sensorial en el sector automovilístico}

Cientos de dólares han sido invertidos en los intentos fallidos de reproducir el olor del Rolls-Royce Silver Cloud de 1965. Para Lindstrom (2005), este olor una pequeña joya del marketing sensorial- es imposible de comprar y ha sido la clave del éxito de esta marca. Cuando Rolls-Royce empezó a recibir quejas sobre los nuevos modelos comparados con los ilustres predecesores, la compañía decidió estudiar la causa de las mismas, concluyendo que la única diferencia existente entre los antiguos modelos y los modernos -aparte de las diferencias técnicas evidentes- era el olor. Con los cambios de las normas de seguridad relativas a los coches, varios materiales utilizados en la fabricación de los modelos de Rolls-Royce se vieron alterados, lo que redundó en un cambio de olor. La única alternativa sería recrear de forma simulada un aroma similar al

REDMARKA UIMA-Universidad de A Coruña - CIECID

Año X, Número 18, (2017), v I pp. 21-47

http://www.redmarka.net/ ISSN 1852-2300 


\section{EL USO DEL NEUROMARKETING Y DEL MARKETING SENSORIAL EN LOS EVENTOS: UN ESTUDIO DE CASO}

Guerra Serrano, Alberto

de los coches antiguos, tarea para la que la compañía dedicó un departamento al completo.

En 2003, Cadillac comenzó a fabricar un aroma artificial para añadir al cuero de los asientos de sus nuevos modelos. El aroma, denominado Nuance, fue creado en laboratorio teniendo en cuenta los resultados conseguidos tras varias encuestas y focus group con clientes de la marca. Es sabido que el cuero usado en los coches de marcas de lujo es procesado con el fin de restarle al máximo el aroma original, añadiéndole a continuación un aroma artificial desarrollado por la marca.

Según Bijsterveld et al. (2014), en la década de 1990 se debatió acerca de cómo debería sonar un coche y se midieron los sonidos para mejorar la experiencia de los usuarios. Se analizaron elementos como el motor, la transmisión, las ruedas, las puertas o los neumáticos buscando una mejora de los resultados en relación con el ruido. Empresas como Chevrolet crearon departamentos exclusivos para este tipo de investigaciones, como fue el caso del departamento de análisis del ruido de las puertas.

Lindstrom (2005) señala que en el mundo de la fabricación de automóviles se han seguido las pautas del diseño organoléptico. Esta técnica incorpora el sabor y el aroma como partes fundamentales del proceso del diseño de un producto y busca la sinergia entre lo que los consumidores saborean y huelen con lo que ven, tocan y escuchan.

Por último, no se puede obviar la publicidad en el sector automovilístico. Según el Estudio Infoadex de la Inversión Publicitaria en España 2014, el sector del automóvil es el tercero en el que más se invierte en publicidad en este país. En los últimos años se ha evolucionado hacia una publicidad más emocional. El ejemplo emblemático es el spot "Mano", de BMW, difundido en el año 2001. En este spot se ve tan solo una mano, que sale de la ventanilla de un coche y ondea al viento, y la marca BMW. Una voz en off pregunta: “¿te gusta conducir?". En ningún momento se enumeran las características técnicas del vehículo, puesto que el spot se centra exclusivamente en evocar la experiencia de conducir. 


\section{EL USO DEL NEUROMARKETING Y DEL MARKETING SENSORIAL EN LOS EVENTOS: UN ESTUDIO DE CASO}

Guerra Serrano, Alberto

\section{Metodología}

El presente estudio fue realizado a partir de una investigación documental acerca de las bases generales del neuromarketing y del marketing sensorial o marketing de experiencias enfocado hacia su posible utilización en los eventos. En el contexto de esta investigación, se considera el evento como un producto con unas características tangibles e intangibles y lanzado para satisfacer las necesidades y deseos de su mercado objetivo (Borges, 2013).

Con el propósito de comprender la aplicación del neuromarketing y del marketing sensorial a un evento y señalar sus características y posibles ventajas, se llevó a cabo el análisis de un evento de carácter promocional realizado en Boadilla del Monte (Madrid, España) el 10 de noviembre de 2015, donde se aplicaron técnicas correspondientes a ambas disciplinas para potenciar las experiencias vividas en el mismo. El acontecimiento consistió en la presentación, a un total de 70 profesionales influyentes de la prensa del motor y del lifestyle, de la nueva gama de vehículos de la marca Suzuki y de su nueva campaña publicitaria, protagonizada por el humorista español Florentino Fernández. Para la realización del estudio de caso y su posterior descripción y análisis, se utilizó la técnica de la observación directa.

En paralelo al estudio de caso y con carácter complementario, se realizó una encuesta personal breve, anónima y de respuestas cerradas a una serie de invitados para conocer sus opiniones acerca del evento. La muestra, compuesta por 12 participantes, no pretende ser representativa, sino una mera aproximación a la percepción y la experiencia de los invitados, ofreciendo un feedback a la organización del evento en cuanto a la efectividad de las técnicas de neuromarketing y marketing sensorial utilizadas. Las preguntas fueron realizadas tras la presentación del director de marketing de la compañía y antes de la salida de los invitados a la prueba de los coches, actividad contemplada en la escaleta del evento. 


\section{EL USO DEL NEUROMARKETING Y DEL MARKETING SENSORIAL EN LOS EVENTOS: UN ESTUDIO DE CASO}

Guerra Serrano, Alberto

\section{Resultados}

\subsection{Estudio de caso}

El evento objeto del presente estudio tuvo lugar en el restaurante La Campana, situado en Boadilla del Monte (Madrid, España), desde las 10:00 hasta las 16:00 horas del martes 10 de noviembre de 2015. El organizador principal fue Ebent Comunicación Integral y el cliente la compañía japonesa del sector automovilístico Suzuki. Durante el evento, se mostraron todos los modelos de la gama relativos a la temporada 2016 , algunos de ellos renovados, como es el caso del Suzuki Vitara.

El escenario del evento fue una recreación de un cine de verano al aire libre. En la gran pantalla se expusieron las características de los nuevos vehículos y los datos correspondientes a la nueva gama, así como la campaña publicitaria protagonizada por el humorista español Florentino Fernández.

La escaleta del evento comenzó con un café de bienvenida. A continuación, los invitados fueron trasladados a la zona del cine de verano, que hasta ese momento permanecía oculta tras unas cortinas para lograr el efecto sorpresa. Una vez inmersos en el escenario recreado, se les ofreció una botella de agua, un paquete de golosinas, una bolsa de palomitas y un perrito caliente ${ }^{3}$. Todos los invitados tuvieron la oportunidad de elegir el coche que fuera de su gusto para vivir la experiencia del cine de verano.

Como colofón de esta primera parte del evento, se les indicó a los participantes una ruta establecida para la prueba de los coches; mientras hacían el recorrido, escuchaban las palabras del presidente de la marca a través de la radio del coche. Al finalizar la ruta, los asistentes regresaron al recinto donde se les hizo entrega de un regalo de agradecimiento y, finalmente, se les invitó a

\footnotetext{
${ }^{3}$ Todos los productos alimenticios llevaban la marca organizadora serigrafiada, una técnica que actúa en el refuerzo y en el recuerdo de la marca.
} 


\section{EL USO DEL NEUROMARKETING Y DEL MARKETING SENSORIAL EN LOS EVENTOS: UN ESTUDIO DE CASO}

Guerra Serrano, Alberto

comer. Con las palabras del director comercial estratégico, del humorista y del presidente de la marca en España se cerró el evento.

A continuación, se enumeran los aspectos más destacados del evento:

- Propuesta: simulación de la experiencia de un cine de verano aplicada a la presentación de una gama de automóviles.

- Duración del evento: mañana y tarde.

- Temporada: otoño (noviembre).

- Desarrollo del evento: distintas etapas organizadas tanto en local cubierto como al aire libre.

- Presencia del protagonista de la campaña publicitaria: contratación de un personaje famoso que se convierte en la imagen de la campaña publicitaria de la nueva gama de Suzuki.

- Organizador del evento: único (Ebent Comunicación Integral).

- Número de vehículos: cuatro ejemplares de cada modelo hasta completar la gama.

- Recursos técnicos: empleo de tecnología de última generación.

De la descripción del evento en sí y de los aspectos señalados se infiere que el evento tenía por objetivo:

- Dar a conocer la nueva gama de automóviles de Suzuki a la prensa especializada.

- Relacionar cada acción y experiencia vivida in situ con la marca Suzuki.

- Mejorar la imagen de marca.

- Estimular el recuerdo de marca.

- Promover el "boca a oreja", objetivo principal del llamado buzz marketing.

- Ofrecer a los invitados una experiencia que estimulara los sentidos.

En relación con el último objetivo, se observa la utilización de técnicas y herramientas de neuromarketing y de marketing sensorial a lo largo del evento, puesto que su planteamiento está enfocado a mejorar las experiencias vividas por los invitados. De acuerdo con Lindstrom (2008) o Braidot (2009), dicho 


\section{EL USO DEL NEUROMARKETING Y DEL MARKETING SENSORIAL EN LOS EVENTOS: UN ESTUDIO DE CASO}

Guerra Serrano, Alberto

planteamiento es acertado debido a que, como se ha indicado anteriormente, la mayoría de las decisiones tomadas por los consumidores provienen del subconsciente y de las partes más internas del cerebro. Una de las maneras más eficaces de motivar una determinada toma de decisiones es a través de la explotación de los sentidos y de las emociones en experiencias como las vividas en el caso que nos ocupa.

Centrándonos en los objetos utilizados para recrear un cine de verano, cabe destacar que se alquilaron equipamientos característicos, como la máquina de palomitas. Esta máquina genera un olor capaz de evocar un cine real. En conjunto con la información olfativa, se estimuló el sentido de la vista, ya que las palomitas eran servidas en un cucurucho con la marca gráfica de Suzuki y sus colores corporativos, reflejados igualmente en el atuendo de las acomodadoras.

El elemento más llamativo fue la gran pantalla ( $17 \times 10$ metros), sujeta por un truss metálico y totalmente visible desde cualquier punto de la sala. El local contaba, además, con un equipo de sonido similar al de los cines al aire libre.

Mediante el sistema de sonido externo y las radios de los coches se hizo uso de la audición. El efecto de escuchar lo que se decía en el exterior a través de la radio del coche se acentuó gracias a los sistemas de insonorización de los vehículos. Por otra parte, el paso de las personas por la moqueta camino de los coches generó un sonido característico de locales como cines o teatros.

El sentido del tacto se estimuló una vez los invitados se encontraban dentro de los vehículos. La textura del volante, de los asientos y de la carrocería trataba de establecer una conexión eficaz entre la experiencia vivida en el evento y el tema automovilístico asociado a la presentación de la nueva gama de Suzuki.

Siendo el tacto un sentido que necesita ser estimulado de forma directa, se considera un acierto invitar a los participantes a subirse a los automóviles y palpar los materiales del interior de los coches. El sentido del gusto se activó con la comida dispuesta para los asistentes. En este punto se percata una actuación colaborativa entre todos los sentidos. 


\section{EL USO DEL NEUROMARKETING Y DEL MARKETING SENSORIAL EN LOS EVENTOS: UN ESTUDIO DE CASO}

Guerra Serrano, Alberto

Por tanto, se observa que los asistentes recibieron estímulos neurosensoriales durante el evento. Se puede afirmar que existe una preocupación por parte de la organización a la hora de motivar el uso de todos los sentidos en mayor o menor medida.

\subsection{Encuesta}

Como se ha explicado, se realizó una encuesta a 12 invitados en la finalización de la etapa anterior a la prueba de vehículos. El cuestionario constaba de cinco preguntas breves relacionadas con el evento y la aplicación de técnicas de neuromarketing y marketing sensorial a un evento promocional, como se expone a continuación.

\section{Gráfico 1: La tecnología empleada ayuda a mejorar el evento}

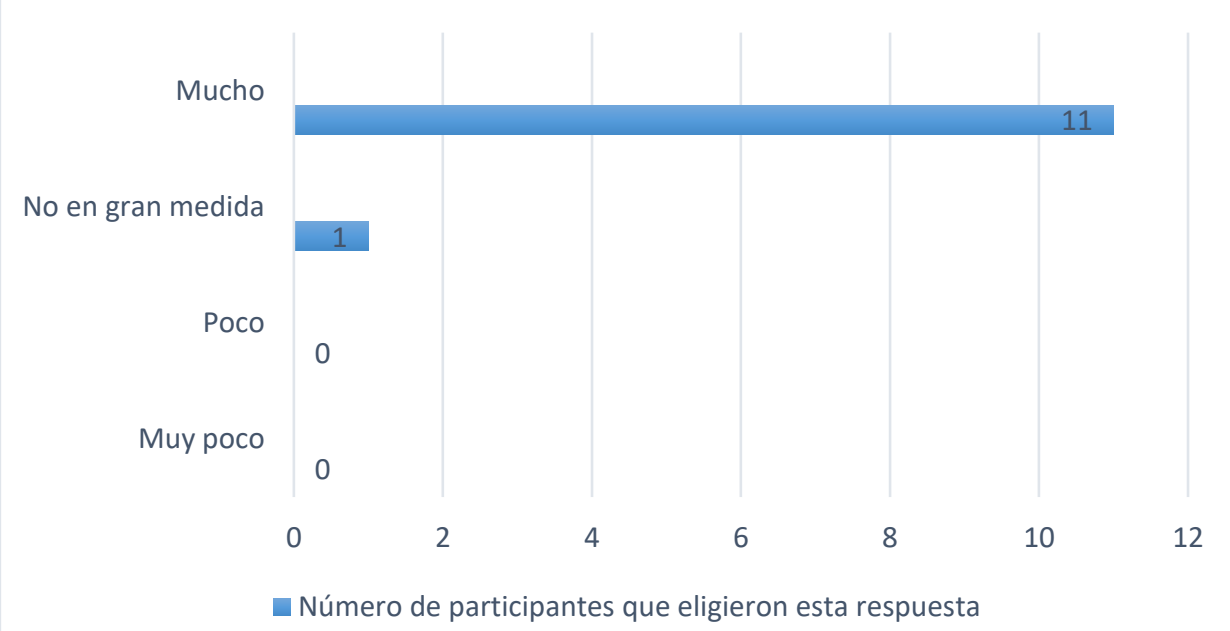

Fuente: elaboración propia.

En primera instancia, se preguntó a los invitados encuestados si creían que la tecnología utilizada en el evento ayudaba a mejorar la experiencia del mismo (véase gráfico 1). En las respuestas obtenidas se aprecia la importancia que toman las tecnologías de última generación en el desarrollo de un evento como el que se analiza. Igualmente, se puede considerar un acierto el uso de las mismas en el evento. 
EL USO DEL NEUROMARKETING Y DEL MARKETING SENSORIAL EN LOS EVENTOS: UN ESTUDIO DE CASO

Guerra Serrano, Alberto

\section{Gráfico 2: Recreación satisfactoria de un cine de verano}

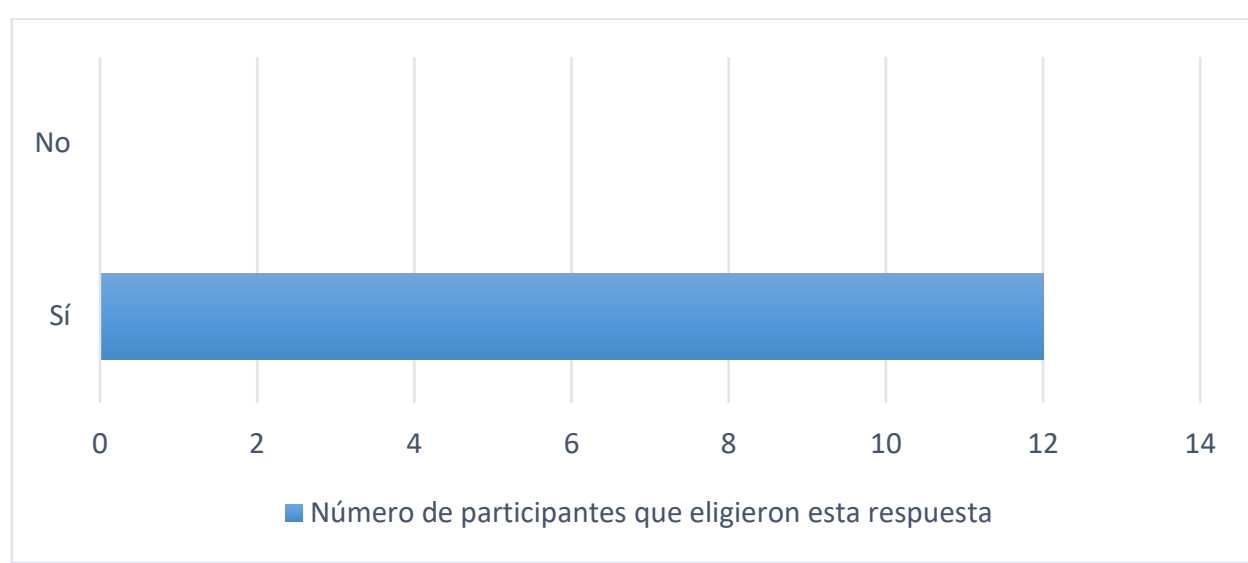

Fuente: elaboración propia.

El gráfico 2 refleja el intento de averiguar si los elementos utilizados durante el evento evocaron a los participantes la sensación de estar en un cine de verano. La unanimidad de las respuestas sostiene la validez y la eficacia de las técnicas de neuromarketing y marketing sensorial utilizadas para recrear el ambiente y la estructura de un cine de verano, estimulando los sentidos de los asistentes.

\section{Gráfico 3: Evaluación del evento}

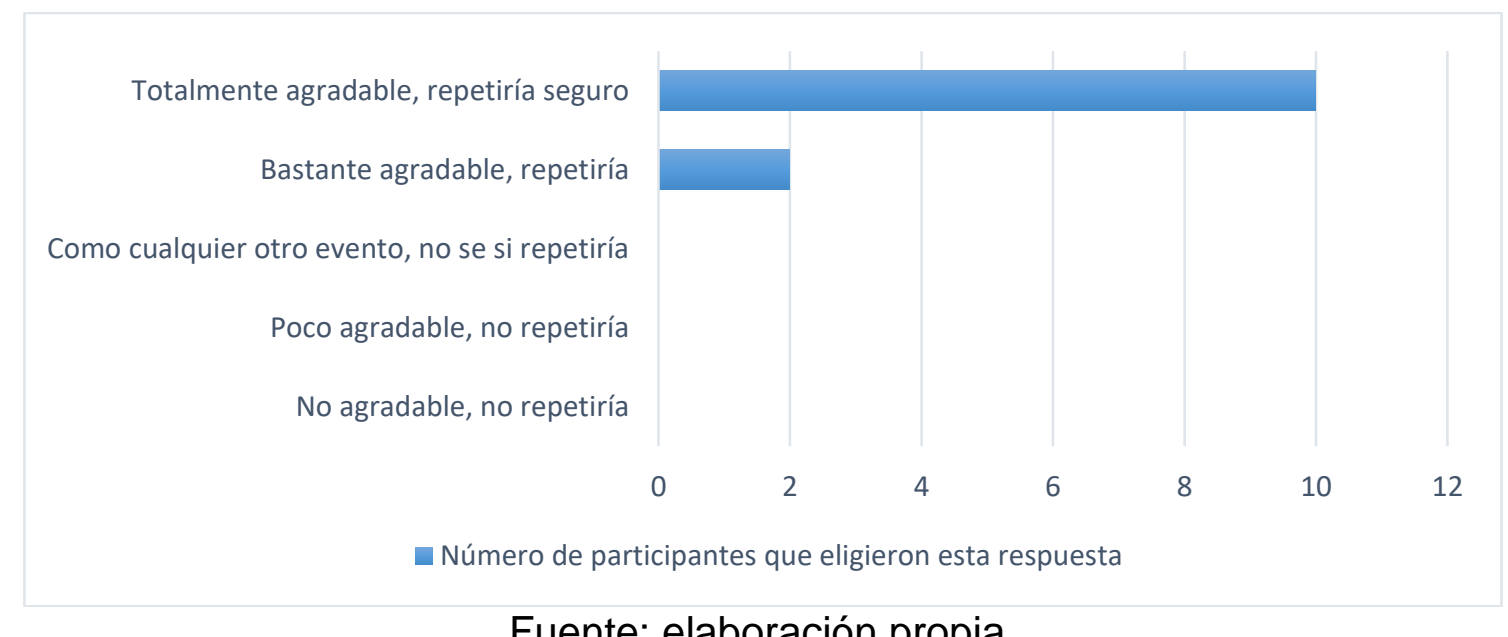

Fuente: elaboración propia. 


\section{EL USO DEL NEUROMARKETING Y DEL MARKETING SENSORIAL EN LOS EVENTOS: UN ESTUDIO DE CASO}

Guerra Serrano, Alberto

Con la pregunta contemplada en el gráfico 3, se buscaba conocer la opinión de los invitados acerca del evento. Se utiliza el parámetro de la agradabilidad respecto a la experiencia vivida con el fin de valorar si esta ha sido lo suficientemente buena como para que los asistentes quisieran repetirla en ocasiones futuras.

Como se puede observar, las respuestas son positivas, lo que apunta hacia el éxito del evento. Experiencias como las descritas en el caso de estudio pueden resultar satisfactorias, promoviendo la fidelidad de los consumidores y mejorando en gran medida las relaciones de los mismos con la marca.

\section{Gráfico 4: Mejora de la imagen de marca de Suzuki}

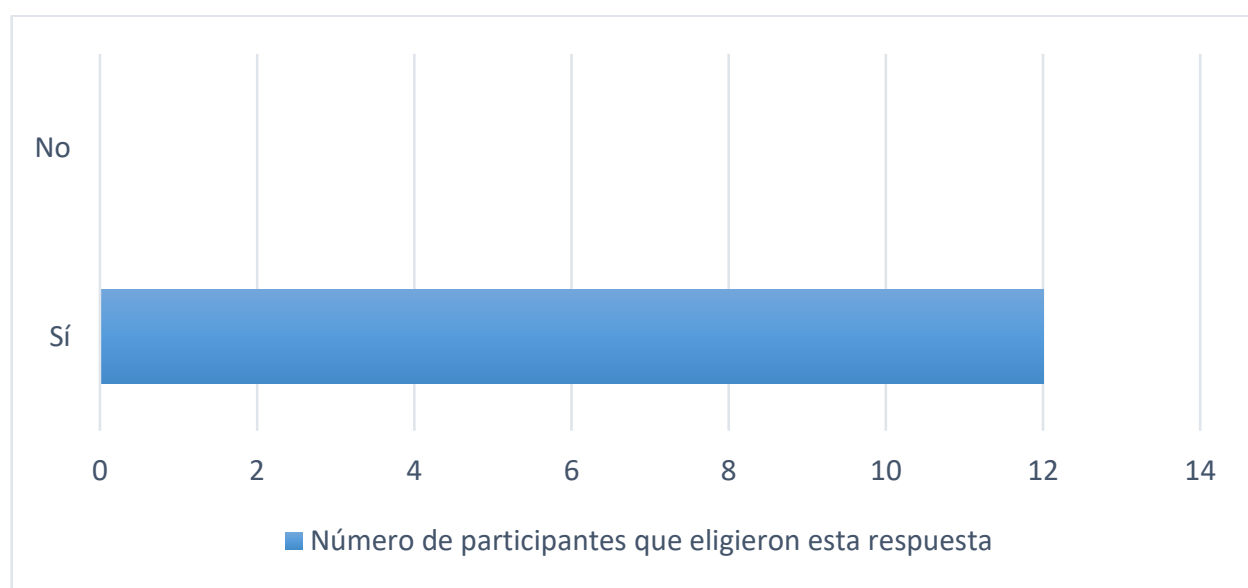

Fuente: elaboración propia.

La siguiente pregunta introduce un elemento que también se trabaja en el evento analizado: la imagen de marca. La unanimidad de las respuestas a la pregunta "¿Cree usted que Suzuki mejora su imagen de marca tras la experiencia que ha vivido?", una vez más, refuerza la idoneidad de las técnicas de neuromarketing y marketing sensorial empleadas en eventos de este tipo (véase gráfico 4).

\section{Gráfico 5: Sentido más estimulado}




\section{EL USO DEL NEUROMARKETING Y DEL MARKETING SENSORIAL EN LOS EVENTOS: UN ESTUDIO DE CASO}

Guerra Serrano, Alberto

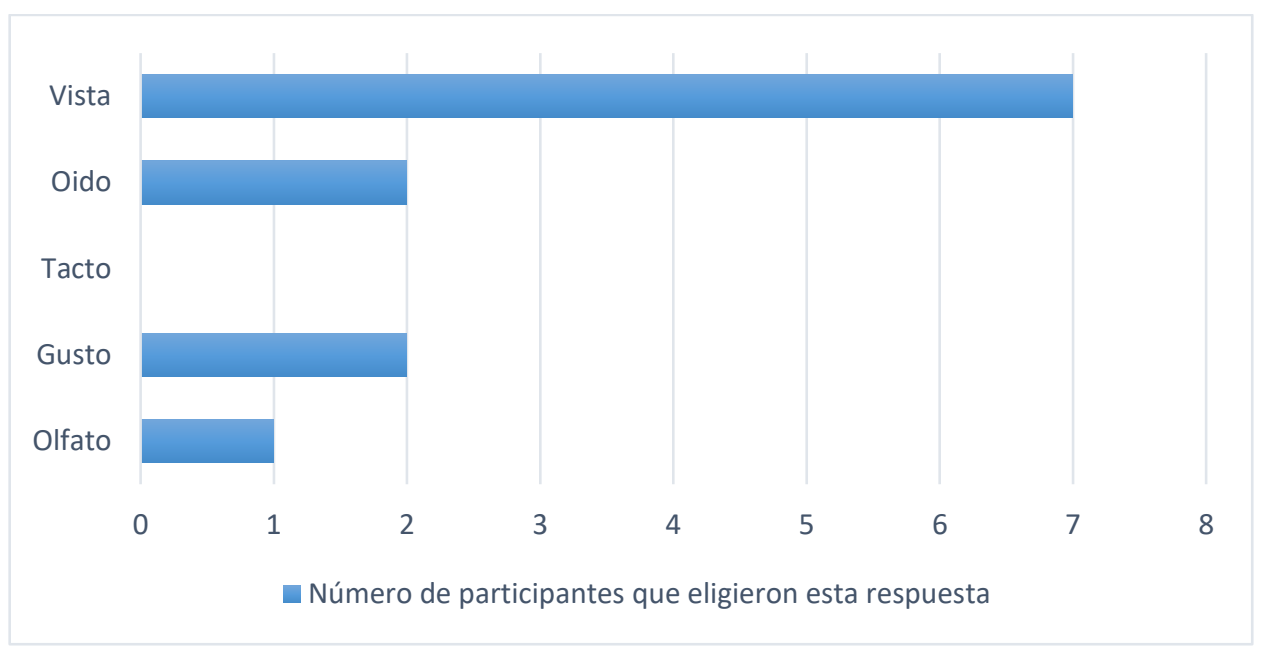

Fuente: elaboración propia.

Por último, se pretendía conocer cuál fue el sentido que más estímulos recibió a lo largo del evento. Las respuestas recogidas en el gráfico 5 indican, por un lado, el predominio de la vista sobre los demás sentidos, puesto que el $58,33 \%$ de los encuestados no dudaron en señalarlo como el sentido más estimulado. Por otro, queda patente la subjetividad de la percepción, ya que el $41,67 \%$ de los entrevistados eligieron otras opciones de respuesta, como el oído, el gusto o el olfato. Llama la atención el hecho de que no se mencionara el tacto, lo que podría indicar una sobrecarga de los demás sentidos en detrimento de la atención que se podría haber dado al tacto de las texturas de los vehículos.

\section{Conclusiones}

En el presente estudio se ha propuesto analizar un evento promocional realizado por la marca Suzuki en la presentación de la gama de vehículos de la temporada 2016 a profesionales de la prensa del motor en España. En dicha ocasión se aplicaron diversas técnicas de neuromarketing y marketing sensorial para enriquecer las experiencias vividas durante el evento, mejorar la imagen de marca y reforzar el recuerdo de la misma por parte de los asistentes. 


\section{EL USO DEL NEUROMARKETING Y DEL MARKETING SENSORIAL EN LOS EVENTOS: UN ESTUDIO DE CASO}

Guerra Serrano, Alberto

Con la observación directa del evento se ha podido realizar un registro en el que se procura recrear tanto los escenarios como las etapas del acontecimiento. En este proceso descriptivo-analítico, se ha hecho hincapié en los principales elementos que pueden ser utilizados para potenciar los sentidos, enlazándolos con las disciplinas del marketing que constituyen la base teórica de este estudio.

Considerando los recursos empleados durante el evento, queda patente la importancia de los intangibles en las estrategias de comunicación, relaciones públicas y marketing. Se observa, por tanto, el valor real y potencial de los implícitos y de las connotaciones relacionadas con la experiencia vivida in situ y las sensaciones provocadas por el uso de técnicas que actúan de manera menos invasiva que la publicidad tradicional. En esta línea, se puede afirmar que el deseo manifiesto de volver a vivir la experiencia que tuvo lugar en el caso estudiado representa una buena labor de comunicación corporativa que podría redundar en un satisfactorio retorno sobre la inversión (ROI), hipótesis que podría verificarse en estudios futuros.

Son reseñables las ventajas del uso de técnicas de neuromarketing y marketing sensorial en la conversión de las distintas sensaciones en experiencias, reflejadas en la proyección de una imagen de marca positiva. Aunque dichas técnicas no son nuevas, es menester ahondar en los efectos de la aplicación de éstas en contextos como los eventos promocionales. El empleo equilibrado de estímulos que alcancen a todos los sentidos de modo que el mensaje sensorial llegue de manera eficaz al público objetivo, de acuerdo con el estudio realizado, constituye uno de los grandes desafíos y quizá la principal asignatura pendiente del marketing de experiencias aplicado a los eventos.

\section{Referencias bibliográficas}

Abril, C., Gavilán, D. \& Sierra, T. (2011). Marketing olfatorio: el olor de los deseos. Marketing y Ventas, 103, pp. 34-39. 
EL USO DEL NEUROMARKETING Y DEL MARKETING SENSORIAL EN LOS EVENTOS: UN ESTUDIO DE CASO

Guerra Serrano, Alberto

Álvarez del Blanco, R. (2011). Marca multisensorial: espléndidamente lucida. Harvard Deusto Marketing y Ventas, 106, pp. 26-33.

Balcetis, E. \& Dunning, D. (2006). See what you want to see. Motivational influences on visual perception. Journal of Personality and Social Phychology, 91(4), pp. 612-615.

Bijsterveld, K., Cleophas, E., Krebs, S. \& Mom, G. (2014). Sound and Safe: A History of Listening Behind the Wheel. Estados Unidos: Oxford University Press.

Blakemore, C. (4 de febrero de 2010). Do we want brain scanners to read our minds? The Telegraph, Reino Unido. Recuperado el 21 de octubre de 2016 de https://goo.gl/4BhJRp.

Bloch, P.H., Sherrell, D. \& Ridgway, N. (1986). Consumer Search: An Extended Framework. Journal of Consumer Research, 13, pp.119-126.

Bone, R. \& Ellen, R. (1992). The generation and consequences of communication- evoked imagery. Journal of Consumer Research, 19, pp. 93104.

Borges, A. (2013). La innovación en la organización de eventos. Compé, Revista Científica de Comunicación, Protocolo y Eventos, 1, pp. 46-60.

Boricean, V. (2009). Brief history of neuromarketing. In: ICEA - FAA. The International Conference on Economics and Administration. Faculty of Administration and Business, University of Bucharest, Romania. 
EL USO DEL NEUROMARKETING Y DEL MARKETING SENSORIAL EN LOS EVENTOS: UN ESTUDIO DE CASO

Guerra Serrano, Alberto

Braidot, N. (2009). Neuromarketing. ¿Por qué tus clientes se acuestan con otro si dicen que les gustas tú? Barcelona: Gestión 2000.

Brighthouse Institute for Thought Sciences (2002). Brighthouse Istitute for Thought Sciences Launches First Neuromarketing Research Company. Company Uses Neuroimaging to Unlock the Consumer Mind. Recuperado el 2 de diciembre de 2016 de https://goo.gl/t00Ctv.

Burnkrant, R. \& Cousineau, A. (1975). Informational and Normative Social Influence in Buyer Behavior. Journal of Consumer Research, 2, pp. 206-215.

Celier, P. (2004). Le Marketing Sensoriel. Marruecos: Mohammedia ENSET School.

De Andreis, A. (2012). Neuromarketing: una mirada a la mente del consumidor. Ad-Gnosis, 1(1), pp. 51-57.

Duchowski, A. (2007). Eye Tracking Methodology: Theory and Practice. Londres: Springer.

Hubert, M. \& Kenning, P. (2008). A current overview of consumer neuroscience. Journal of Consumer Behavior, 7, pp. 272-292.

Infoadex (2014). Estudio Infoadex de la Inversión Publicitaria en España. Recuperado el 5 de octubre de 2016 de https://goo.gl/Wytq73.

Interactive Advertising Bureau - Spain (IAB Spain) (2015). III Estudio de Digital Signage de IAB Spain. Recuperado el 10 de octubre de 2016 de https://goo.gl/vErPVR. 


\section{EL USO DEL NEUROMARKETING Y DEL MARKETING SENSORIAL EN LOS EVENTOS: UN ESTUDIO DE CASO}

Guerra Serrano, Alberto

Kenning, P., Deppe, M., Schwindt, W., Kugel, H. \& Plassmann, H. (2009). The good, the bad and the forgotten. An fMRI study on ad liking and ad memory. Advances in Consumer Research, 36, pp. 4-7.

Krishna, A., Elder, R.S. \& Caldara, C. (2010). Feminine to smell but masculine to touch? Multisensory congruence and its effect on the aesthetic experience. Journal of Consumer Psychology, 20(4), pp. 410-418.

Lee, N., Broderick, A.J. \& Chamberlain, L. (2007). What is 'neuromarketing'? A discussion and agenda for future research. International Journal of Psychophysiology, 63(2), pp. 199-204.

Lewis, D. \& Bridger, D. (2005). Market Researchers make Increasing use of Brain Imaging. Advances in Clinical Neuroscience and Rehabilitation, 5(5), pp. 36-37.

Lindstrom, M. (2005). BRAND sense. Building Powerful Brands through Touch, Taste, Smell, Sight y Sound. Estados Unidos: Simon y Schuster.

Lindstrom, M. (2008). Buyology: Truth and Lies About Why We Buy. Estados Unidos: Doubleday.

Manzano, R., Avello, M. \& Abril, C. (2011). Marketing visual: amor a primera vista. Marketing y Ventas, 103, pp. 46-51.

Manzano, R., Gavilán, D., Avello, R., Abril, C. \& Serra, T. (2012). Marketing Sensorial. Comunicar con los sentidos en el punto de venta. Madrid: Pearson Educación.

Matthews, D. (2008). Special Event production. The resources. Reino Unido: Butterworth-Heinemann. 
EL USO DEL NEUROMARKETING Y DEL MARKETING SENSORIAL EN LOS EVENTOS: UN ESTUDIO DE CASO

Guerra Serrano, Alberto

Morrin, M., Krishna, A. \& Lwin, M.O. (2011). Is Scent-Enhanced Memory Immune to Retroactive Interference? Journal of Consumer Psychology, 21, pp. 354-361.

Petrova, P. \& Cialdini, R. (2005). Fluency of consumption imagery and the backfire effects of imagery appeals. Journal of Consumer Research, 32(3), pp. 442-452.

Pradeep, A.K. (2008). The buying brain. Nueva Jersey: John Wiley y Sons, Inc.

Rieunier, S. (2002). Le marketing sensoriel du point de vente. Recherce et Applications en Marketing, 17(4), pp.46-65.

Schmitt, B. (1999). Experiential marketing: How to get customers to sense, feel, think, act and relate to your company and brands. Nueva York: The Free Press.

Sebastian, V. (2013). Neuromarketing and neuroethics. Social and Behavioral Sciences, 127, pp. 763-768.

Simons, D. \& Chabrics, C.F. (1999). Gorillas in our midst: sustained inattentional blindness for dynamic events. Perception, 28(9), pp.1059-1074.

Spangenberg, E., Crowley, A. \& Henderson, P. (1996). Improving the store environment. Do olfactory cues affect evaluations and behaviors? The Journal of Marketing, 60(2), pp. 67-80.

Tauber, E. (1972). Why do people shop? The Journal of Marketing, 36(4), pp. 644-656. 
EL USO DEL NEUROMARKETING Y DEL MARKETING SENSORIAL EN LOS EVENTOS: UN ESTUDIO DE CASO

Guerra Serrano, Alberto

Zucco, G.M. (2003). Anomalies in cognition: Olfactory memory. European Psychologist, 8(2), pp. 77-86. 